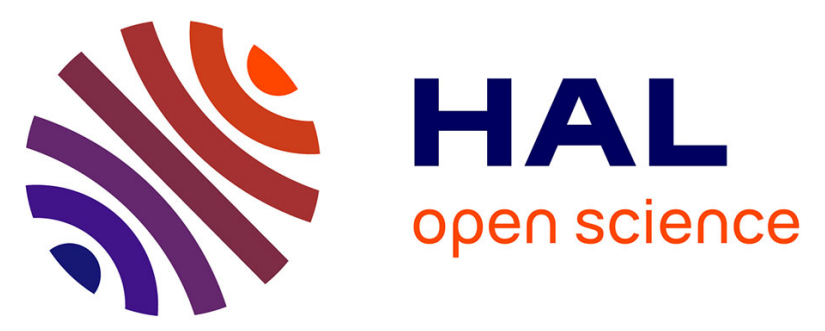

\title{
Seasonal and co-seismic velocity variation in the region of L'Aquila from single station 1 measurements and implications for crustal rheology 23 AUTHORS
}

Piero Poli, Valenting Marguin, Qingyu Wang, Nicola d'Agostino, Paul A. Johnson

\section{To cite this version:}

Piero Poli, Valenting Marguin, Qingyu Wang, Nicola d'Agostino, Paul A. Johnson. Seasonal and coseismic velocity variation in the region of L'Aquila from single station 1 measurements and implications for crustal rheology 23 AUTHORS. Journal of Geophysical Research: Solid Earth, 2020, 125 (7), pp.e2019JB019316. 10.1029/2019JB019316 . hal-02929924

\section{HAL Id: hal-02929924 \\ https://hal.univ-grenoble-alpes.fr/hal-02929924}

Submitted on 4 Sep 2020

HAL is a multi-disciplinary open access archive for the deposit and dissemination of scientific research documents, whether they are published or not. The documents may come from teaching and research institutions in France or abroad, or from public or private research centers.
L'archive ouverte pluridisciplinaire HAL, est destinée au dépôt et à la diffusion de documents scientifiques de niveau recherche, publiés ou non, émanant des établissements d'enseignement et de recherche français ou étrangers, des laboratoires publics ou privés. 


\title{
Seasonal and co-seismic velocity variation in the region of L'Aquila from single station measurements and implications for crustal rheology
}

\author{
AUTHORS: Piero Poli ${ }^{1}$, Valenting Marguin ${ }^{2}$, Qingyu Wang ${ }^{1}$, Nicola D'Agostino ${ }^{3}$, Paul \\ Johnson $^{4}$ \\ 1- Institut de Sciences de la Terre, Université Grenoble Alpes, CNRS (UMR5275), \\ Grenoble, France. \\ 2- Département des sciences de la Terre, Université de Genève, 1205 Geneva, \\ Switzerland \\ 3- Osservatorio Nazionale Terremoti, Istituto Nazionale Geofisica Vulcanologia, Roma, \\ Italy \\ 4- Geophysics, Los Alamos National Laboratory, Los Alamos, New Mexico USA 87545
}

\begin{abstract}
We performed time lapse measurements of velocity variations using empirical Green's functions reconstructed by autocorrelation of seismic noise recorded during a period of 17 years in the region of l'Aquila, Italy. The time lapse approach permitted us to evaluate the spatial (depth) dependence of velocity variation (dv/v). By quantitatively comparing the 17 years of $d v / v$ time series with independent data (e.g. strain induced by earthquakes, hydrological loading) we unravel a group of physical processes inducing velocity variations in the crust over multiple time and spatial scales. We find that rapid shaking due to three magnitude 6+ earthquakes mainly induced near surface velocity variations. On the other hand, Slow strain perturbation (period 5 years) associated with hydrological cycles, induced velocity changes primarily in the middle-crust. The observed behavior suggests the existence of a large volume of fluid filled cracks exist deep in the crust. Our study, beyond shedding new light into the depth dependent rheology of crustal rocks in the region or l'Aquila, highlights the possibility of using seasonal and multiyear perturbations to probe the physical properties of seismogenic fault volumes.
\end{abstract}

\section{Introduction}

Detailed laboratory protocols exist to estimate how rocks respond to strain perturbations, and show that a variety of non-linear responses exists for variable rock types with different physical properties (e.g. cracks density, microstructure, presence of fluid, temperature and pressure effects - Guyer \& Johnson, 1999;2009; Ostrovsky and Johnson, 2001; Renaud et al., 2009, 2012, Riviere et al., 2015).

One approach to describing nonlinear elastic and plastic properties of rock is applying 'effective viscosity' (e.g. Lyakhovsky et al., 2001, Ben-Zion, 2008). Numerical simulations (Lyakhovsky et al., 2001, Hamiel et al., 2006; Lott et al.2018) show how this effective parameter plays a fundamental role in how rocks respond to strain perturbation, and thus controls phenomena occurring during the seismic cycle (e.g. clustering of seismicity, foreshocks, style of nucleation, amount of aseismic slip). The effective viscosity parameter has similarities to the hysteretic nonlinear parameter in the Priesach-Mayergoyz description of elasticity (McCall and Guyer 1993; Guyer and Johnson 2009). The latter has a direct link with damage intensity (e.g., Guyer and Johnson 1999; Johnson 1998, Van Den Abeele and Visscher, 2000, Ostrovsky and Johnson 2001). Other models that describe these behaviours, as well including Ahrennius approaches for hysteresis, exists (e.g., Ostrovsky et al., 2019; Sens-Schoenfelder et al., 2018).

It is thus fundamental to characterize the physical properties of rocks surrounding seismogenic faults, to better understand the role of rheology and elasticity during the 
seismic cycle, and associated phenomena that can arise in fault(s). Field observations emulating laboratory protocols have been attempted, by studying velocity variations due to strong, surface active-source induced shaking (e.g., Johnson et al., 2009), as well as rocks subjected to cycles of tidal forcing and induced seismicity (e.g., Delorey et al, 2017; van der Elst et al, 2017 and active seismic experiments probing the effects of Earth tides (e.g. Yamaura et al., 2003). More recently, the ability of estimating the Green's function from seismic noise (Shapiro \& Campillo, 2004), has made it possible to monitor velocity variations (e.g. Brenguier et al., 2014) without the need of active sources (e.g. explosions; vibrators), and more recent studies applied this method to study the response of rocks to tidal strain (Takano et al., 2014, Hillers et al., 2015b). However, at present only the shallowest portions of the crust (primarily less than $1 \mathrm{~km}$ of depth) have been explored (Takano et al., 2014, Hillers et al., 2015b) and at most, depths to $5 \mathrm{~km}$, following the 2004 M6.0 Parkfield earthquake ( $\mathrm{Wu}$ et al., 2017), thus permitting the characterization of shallow damage zones, but not deeper regions, where the most seismicity occurs and large earthquakes nucleate (see Ben-Zion, 2008, and reference therein).

It has been demonstrated that detailed lapse-time monitoring applying coda waves reconstructed from correlation of seismic noise can provide important information about the deeper part of the crust (Obermann et al., 2013, Obermann et al., 2014, Hillers et al., 2018). These results have been validated with numerical experiments, providing clues regarding the depth resolution of velocity variation $(\mathrm{dv} / \mathrm{v})$ as function of coda lapse-time (Obermann et al., 2013).

In this work, we performed time lapse monitoring of dv/v near to the city of L'Aquila, in the central Apennines (Italy). This region is characterized by active normal faulting that accommodates the ongoing $\sim 3 \mathrm{~mm} / \mathrm{yr}$ tectonic extension (D'Agostino, 2014, Fig. 1). As a result, normal faults capable of producing magnitude $6+$ events are present along the entire Apennine chain. The region of L'Aquila itself is characterized by significant seismic hazard, and was recently struck by 3 magnitude $6+$ earthquakes (http://iside.rm.ingv.it, Fig. 1).

We here estimate the spatial (depth) response $(\mathrm{dv} / \mathrm{v})$ of the medium to periodic perturbations. Instead of relying of tidal strain as has been applied by others (e.g., Hillers et al., 2015), we exploit periodic perturbations associated with multiyear-long hydrological cycle related to the recharge of karst aquifers (e.g. Silverii et al., 2019). These perturbations provide significant strain at the surface (relative baseline elongation up to 10-6 mm within few tens of kilometers, mostly horizontal) with poorly resolved extension at depth. Correlation with seismicity rate in the 1.2-3.9 magnitude range in the southern Apennines (D'Agostino et al., 2019) suggests that stress perturbations induced by the hydrological forcing may extend to a depth larger than $5 \mathrm{~km}$ and thus affect the seismogenic portion of the crust (Fig. 1). In particular, we assess how dv/v over different time windows, evolves for episodes of dilatation and compression of the crust (Silverii et al., 2019). We further estimate the response of the medium to earthquakes $(M>6)$ that occurred during the study period (2000-2016).

In the following, we begin by describing the procedures to derive estimates of the Green's function and measure velocity variations (sec. 2). We then analyze evolution of $\mathrm{dv} / \mathrm{v}$ for periodic perturbation and during coseismic periods (sec. 3 and 4). Finally, we discuss the results and the peculiar evolution of $\mathrm{dv} / \mathrm{v}$ as a function of lapse-time that we observed (sec. 5). Our results suggest that an isolated region extended into the middlelower crust is particularly sensitive to deformations. We interpret this observation as due to the presence of significant amounts of fluid filled cracks at depth that make the material more susceptible to the nonlinear elastic changes we observe. 


\section{Seismic noise correlation and time lapse velocity variation}

\section{a. Estimation of the Green's function from noise correlation}

We calculate the three-component (ZZ, EE, NN) autocorrelation using continuous seismic data recorded at the AQU station located in central Italy (Fig. 1). We focus on the time period from January 12000 to December 312017 (17 years). Each daily trace of seismic data is split in $10 \mathrm{~min}$ windows with $50 \%$ overlap, after the deconvolution of the instrumental response and filtering between 0.5 and $1 \mathrm{~Hz}$. After analyzing several frequency bands, we choose to focus on $0.5-1 \mathrm{~Hz}$ range as it provides the highest quality correlation, while limiting the amount of stacked days, thus increasing the time resolution of our analysis.

To remove spurious spikes and contributions from earthquakes, we calculate the sum of the squared signal (energy) for each window, and remove those exceeding the daily mean of the energy plus 3 standard deviations (Poli et al., 2012). We ensure that, after this test, at least 20 hours of data are still contained in the daily trace; otherwise the daily trace is rejected. The time windows passing the amplitude test are one-bit normalized to further reduce transient signals (e.g. earthquakes), which may escape our processing.

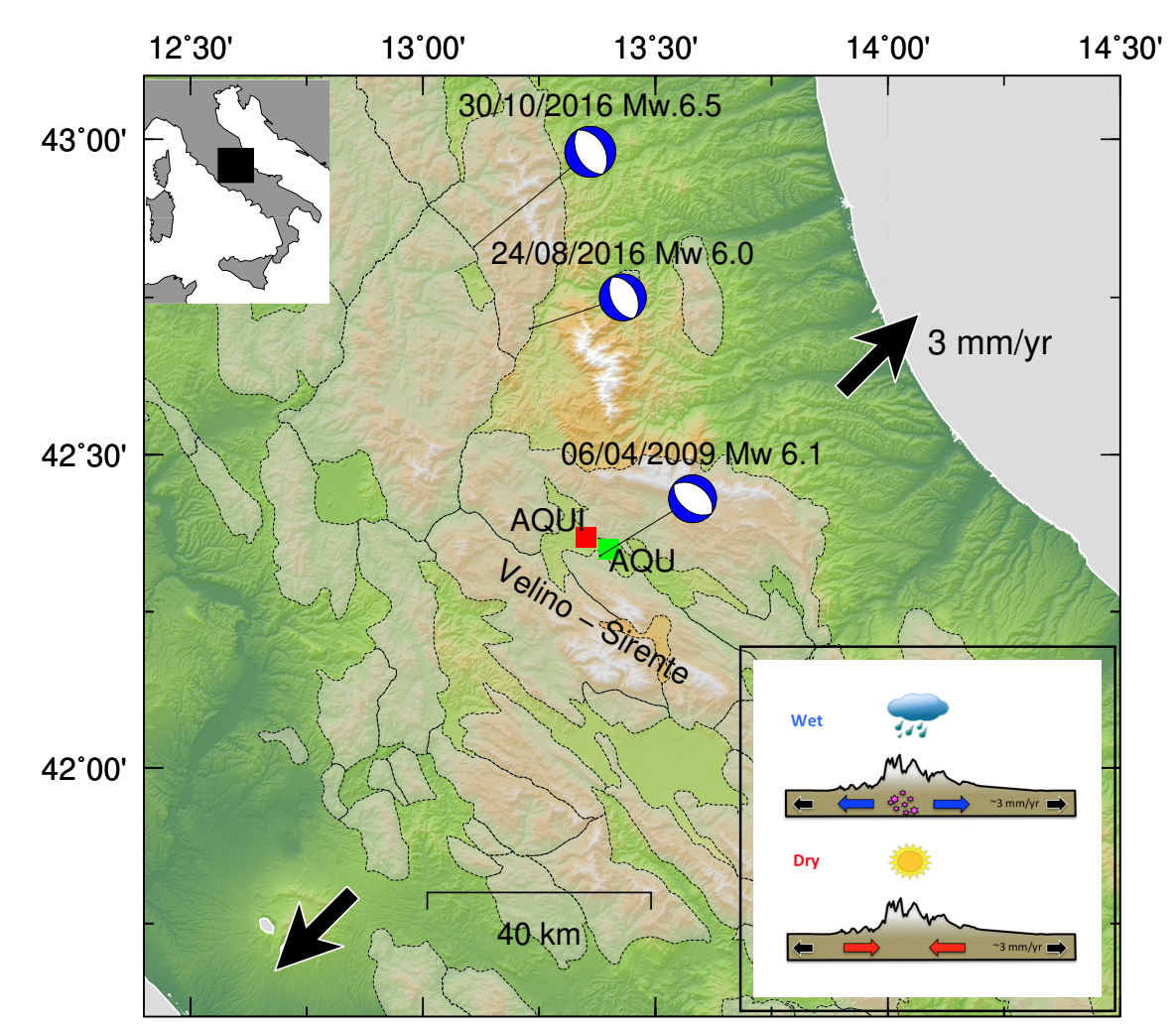

Figure 1. Map of the study area. The upper inset shows the location of the study area in Italy. The squares indicate the location of the seismic (AQU, in green) and GPS (AQUI, in red) stations. Beach balls show focal mechanism of the main events from 2009 to 2016 (from Scognamiglio et al., 2006). Shaded areas include regions where carbonate lithologies host large karst aquifers (see Silverii et al., 2019). The lower inset shows a conceptual drawing of the modulation of crustal deformation and seismicity by seasonal and multi-annual recharge/discharge phases of of karst aquifers. 


\section{b. Measurement of velocity variation (dv/v)}

We measure the velocity variations using both stretching (Lobkis \& Weaver, 2003) and doublet (Poupinet et al., 1984) techniques. While the two methods provide similar results (Fig. S3), additional tests have shown that the stretching technique provides less noisy measurements, and therefore will be used here.

In detail, the velocities are obtained by stacking 90 days using a 89 day overlap of correlations, to ensure stable dv/v measures (Sabra et al., 2005). Each stacked correlation is then compared, using the methods mentioned above, with a reference signal (the stack of the correlation over the full-time period) to estimate the $\mathrm{dv} / \mathrm{v}$ over different coda waves lapse-time (table 1). We then average the $\mathrm{dv} / \mathrm{v}$ over different components, weighting with squared correlation coefficients estimated after stretching. Only signals with correlation larger than 0.9 are retained, and a stack is performed if all the 3 components are available.

\section{c. Lapse time and depth sensitivity of $d v / v$}

In this section, we estimate the first order depth resolution for our velocity variation measures. It should be kept in mind however, that the sensitivity to absolute depth requires detailed measures of the scattering properties of the medium, which are not available for the study area (Obermann et al. 2013; 2016, Kanu and Snieder, 2015). Nevertheless, we can reason around existing theoretical and numerical results to gain insights about relative depth resolution of coda waves measured at different lapse time of the coda (Obermann et al. 2013; 2016, Kanu and Snieder, 2015).

Despite the depth sensitivity of coda waves has not been fully quantitatively solved yet, it is well known that the sensitivity to $\mathrm{dv} / \mathrm{v}$ in a medium varies with frequency and time lapse of the coda waves used (i.e., deeper sampling for larger lapse-time) (e.g. Pacheco \& Snieder, 2005, Obermann et al., 2013, Wu et al., 2017). Numerical simulations (Obermann et al. 2013,2016$)$ reported that the sensitivity of coda waves is due to a combination of the sensitivities of body waves and surfaces waves. In the early normalized lapse-time, $t$ less than $\sim 6\left(t\right.$ is normalized by mean free time $\left.t^{*}\right)$, the coda wave sensitivity is controlled by the surface wave sensitivity. With increasing lapse-time, the body wave sensitivity becomes progressively more important.

We thus start by estimating the sensitivity of surface waves (Hermann, 2013), using a local velocity model (Chiaraluce et al., 2009) and a frequency of $0.75 \mathrm{~Hz}$ (Fig. 2b). The resulting surface wave kernel suggest sensitivity in the uppermost $4 \mathrm{~km}$ of the crust.

We furthermore evaluate the sensitivity of the scattered body waves by considering a 3-D sensitivity kernel formulation by Pacheco and Snieder (2005). The energy propagator $p$ is calculated by the radiative transfer solution approximation for isotropic scattering (Paasschens, 1997; Planès et al., 2014). We estimate the theoretical depth sensitivities of the body wave velocity changes with scattering mean free paths from $10 \mathrm{~km}$ and $100 \mathrm{~km}$, proxies for the high and low frequency regimes (Lacombe et al., 2003, Hiller at al., 2019). The energy velocity is determined by the equipartition state. We take the theoretical equipartition ratio as 10.4 and 3 for a Poisson solid (Margerin et al., 2000; Weaver, 1982), the same as Wang et al. (2019), for further calculation. We solved for the body wave depth sensitivity normalized to $30 \mathrm{~km}$ depth with each layer $1 \mathrm{~km}$ thick layer.

Figure 2a gives the calculated normalized body wave kernel. The calculation follows the details described by Obermann et al., (2013), Planès et al. (2014), Obermann et al. (2016), and Wang at al., (2019). We clearly observe that using the mean free path (1) equal to $100 \mathrm{~km}$ (low frequency regime), the depth sensitivity is deeper than using the smaller mean free path 1 as $10 \mathrm{~km}$. This is an indication of the frequency-dependent depth 
sensitivity of body waves, which is the same characteristic surface waves exhibit (Fig. 2a). We also observe how the depth sensitivity increases gradually as the lapse-time becomes progressively larger from $20 \mathrm{~s}$ to $50 \mathrm{~s}$ with the same mean free path. Thus, we measure the seismic velocity changes for deeper strata applying later lapse-times (Fig. 2a). This result, suggest that by observing the time-lapse evolution of $\mathrm{dv} / \mathrm{v}$ across the coda, we can get insights about velocity variation into deep layers. A similar conclusion was draw by Obermann et al. (2013) by using numerical modeling.

Guided by the calculated kernels and results from previous studies (Obermann et al. 2013), we defined a set of windows (table 1) to scan different depths of the crust, similarly to previous studies based on autocorrelation (e.g. Ricther et al., 2014). In more detail, we defined 3 starting times $(10,20$, and $30 \mathrm{~s}$ ) after zero time (ballistic waves arrival time). For each starting time, we vary the length of the time window to perform stretching $(20,40$, $60 \mathrm{~s})$. The goal of this coda lapse-time dependent analysis is to resolve any evolution of $\mathrm{dv} / \mathrm{v}$, which can reveal the linear and nonlinear elastic response as function of relative depth (e.g. increment of $\mathrm{dv} / \mathrm{v}$ with coda lapse time will highlight larger velocity changes at depth).

Table 1: Time lapse for calculation of $d v / v$.

\begin{tabular}{|l|l|l|}
\hline Window number & Time start (s) & Time end (s) \\
\hline $\mathbf{1}$ & 10 & 30 \\
\hline $\mathbf{2}$ & 10 & 50 \\
\hline $\mathbf{3}$ & 10 & 70 \\
\hline $\mathbf{4}$ & 20 & 40 \\
\hline $\mathbf{5}$ & 20 & 60 \\
\hline $\mathbf{6}$ & 20 & 80 \\
\hline $\mathbf{7}$ & 30 & 50 \\
\hline $\mathbf{8}$ & 30 & 70 \\
\hline $\mathbf{9}$ & 30 & 90 \\
\hline
\end{tabular}
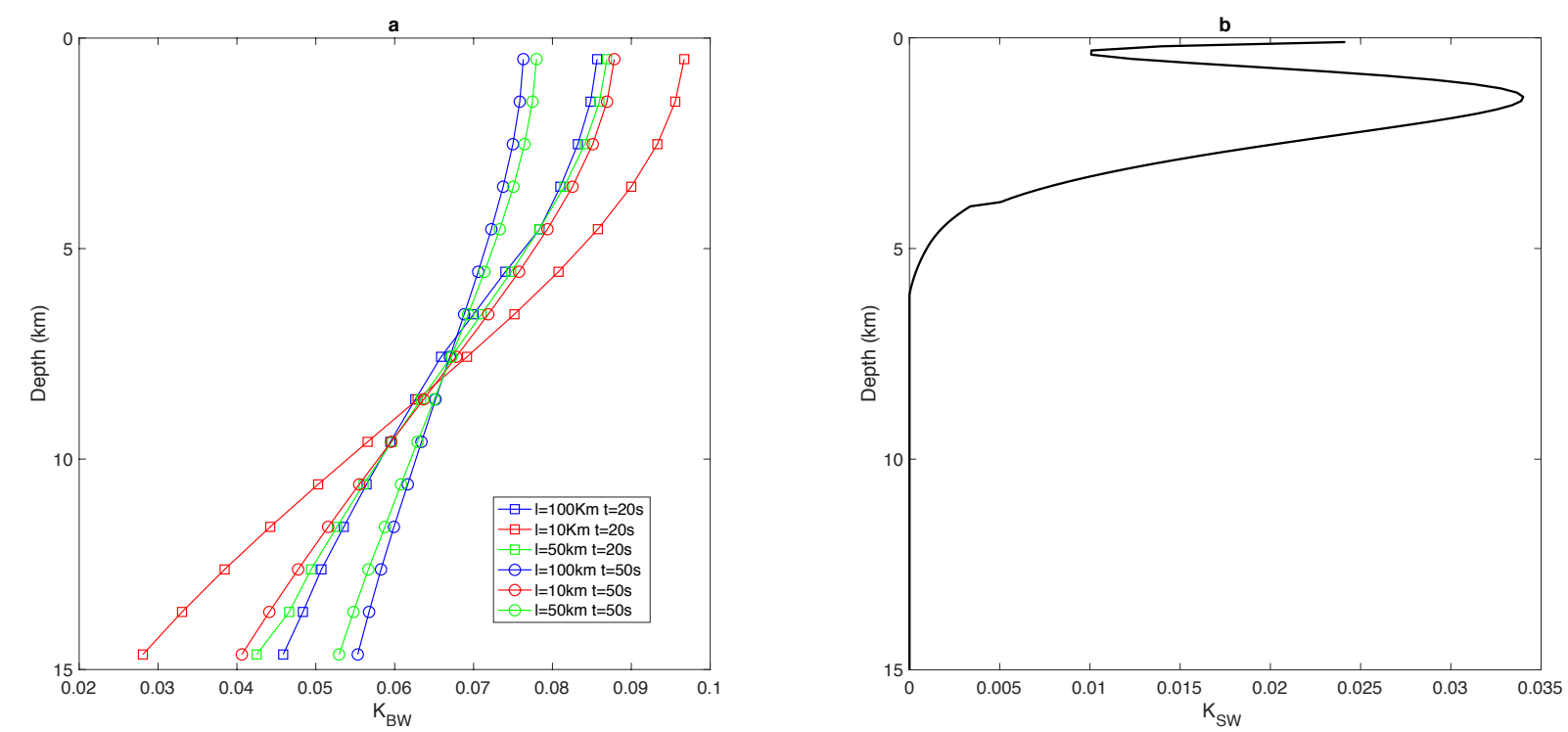

Figure 2: a) Body waves sensitivity kernels for variable mean free paths lapse-times. b) Surface waves sensitivity kernel.

d. Comparison of $d v / v$ with other estimates 
The velocity variation $(\mathrm{dv} / \mathrm{v})$ time series for window 4 (20s to $40 \mathrm{~s}$ lag time) is shown periodic and co-seismic perturbations respectively). Two primary velocity decreases are observed. The largest decrease (relative $\mathrm{dv} / \mathrm{v} \sim 0.3 \%$ ) is associated with the occurrence of the magnitude 6.3 L'Aquila earthquake (iside.rm.ingv.it). The second decrease (relative $\mathrm{dv} / \mathrm{v} \sim 0.15 \%$ ) occurred in 2016 during the Norcia-Visso seismic sequence (Chiaraluce et al., 2016), which includes $2 \mathrm{M} 6+$ earthquakes. Besides of these major coseismic drops, we observe cyclic variations of $\mathrm{dv} / \mathrm{v}$ up to $0.05 \%$ (fig. $3 \mathrm{~b}$ ).

Silverii et al. $(2016,2019)$ analyzed GPS data in the study region, showing the presence of transient deformations associated with multiyear hydrological cycles ( $\sim 5 y r s)$. During these cycles the crust undergoes significant extension and compression (up to $3 \mathrm{~mm} / \mathrm{yr}$ ) sustained for 2-3 years associated, respectively, with phases of recharge and discharge of karst aquifers that modulate the secular, tectonic strain accumulation (see inset Fig. 1). These cycles are visible in both the rain time series (shown as detrended cumulative rain in fig. 3c) and in the detrended horizontal GPS motion (fig. 3e) recorded at the station AQUI (fig. 1). Here we use the east-component time series of the AQUI site corrected for longterm trend and instrumental offsets (see Silverii et al., 2019 for details of the processing).

In periods of significant aquifer recharge (e.g. 2005 to 2007) the AQUI site is subject to a significant eastward displacement $(\sim 5 \mathrm{~mm})$, while the opposite motion is recorded for dry periods. As reported by Silverii et al (2019) the multi-seasonal, hydrological forcing associated to the recharge of karst aquifers induces a measurable surface deformation up to $\sim 90$ nanostrain/yr. The AQUI station is mostly sensitive to the large Velino-Sirente karst system (Fig. 1) alternatively moving to the north-east during phases of high recharge and to the south-west during phases of low-recharge (Silverii et al., 2019). This motion is superimposed on the steady SW-NE $3 \mathrm{~mm} / \mathrm{yr}$ extension across the Apennines. It is important to note that this phenomenon is pervasively observed along the Apennines at stations located close to main karst aquifers (Silverii et al., 2016, 2019; D'Agostino et al., 2018).

Similarly, and in agreement with previous studies (e.g. Hillers et al., 2015a, Wang et al. 2017), the $\mathrm{dv} / \mathrm{v}$ follows the hydrological cycle, with a velocity drop for increasing hydraulic head and vice versa. The dv/v also shows shorter term oscillations ( $\sim 1 \mathrm{yr})$ which are visually correlated with the variation of surface temperature (fig. 3d). In agreement with previous studies (e.g. Richter et al., 2014) a decrease in temperature coincides with a reduction of dv/v. Alternatively, these, short-term oscillations can be due to short term seasonal hydrological effects similar to what is observed in the GPS time series (Silverii et al., 2016).

In the remainder of this work we will quantify the response of the crust to periodic (hydrological) and tectonic (earthquakes) forcing and assess the sensitivity of different crustal levels to these deformations. 


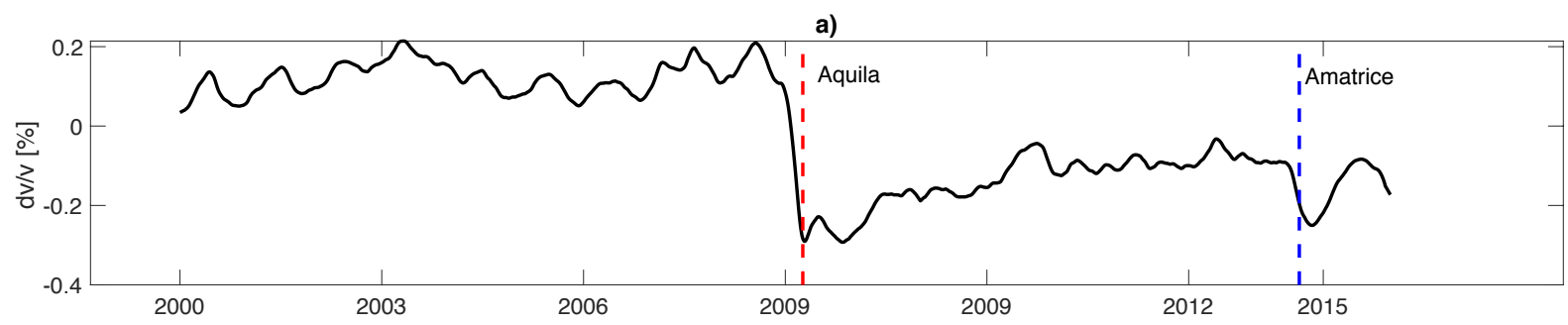

b)
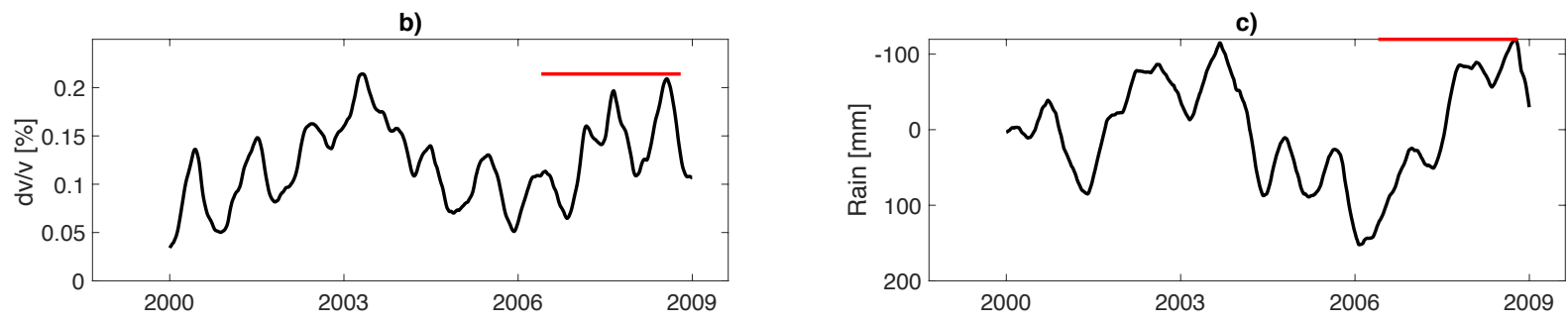

d)
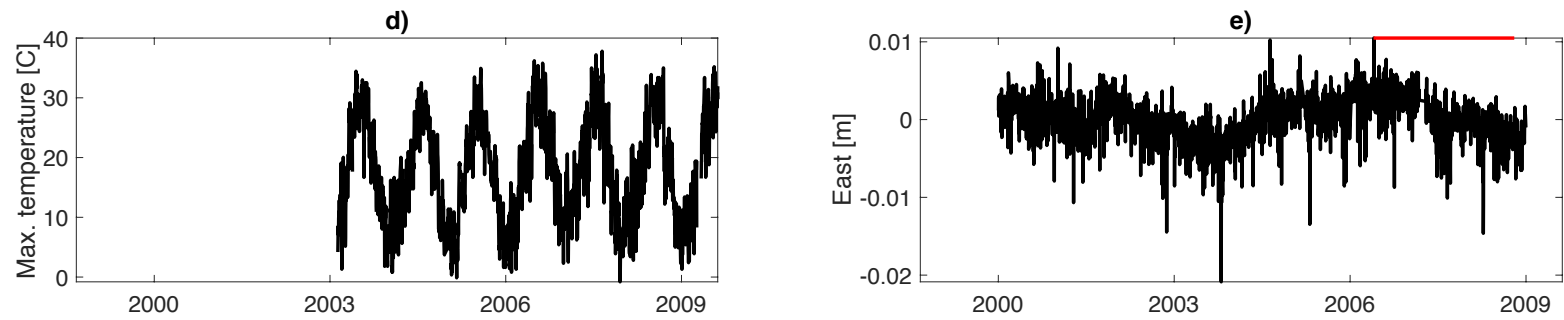

FIGURE 3: Summary of observations in the study area. a) Time series of velocity variation for the entire period, with major earthquakes highlighted. b) $d v / v$ time series during the preseismic period. c) Detrended cumulative rain as in Silverii et al. (2019) (y-axis is inverted). d) Daily maximum temperature. e) East GPS displacement at station AQUI corrected for tectonic trend and antenna offset from Silverii et al.(2009). The red solid line indicate a dry period identified by Silverii et al.(2009).

\section{Sensitivity to cyclic deformations and probing the mid-crust}

\section{a. Decomposition of $d v / v$ time series}

We quantitatively evaluate the seasonal and multi-years components of $\mathrm{dv} / \mathrm{v}$ estimated for different lapse times (see table 1). In this section, we focus only on the pre-seismic period, using dv/v measured before L'Aquila earthquake (beginning of 2000 to end of 2008).

We start by modeling the relative contribution of the multiyear and seasonal effect on $\mathrm{dv} / \mathrm{v}$ with a phenomenological model (e.g. Taira et al, 2018):

$$
\frac{\mathrm{dv}}{\mathrm{v} S}=\mathrm{A}+\mathrm{B} \cos \left(\frac{2 \pi}{\mathrm{C} * 365}(\mathrm{t}-\mathrm{D})\right)+\mathrm{E} \cos \left(\frac{2 \pi}{\mathrm{F} * 365}(\mathrm{t}-\mathrm{G})\right)[2]
$$

In eq. 2, $\mathrm{A}$ is a global offset, $\mathrm{B}$ and $\mathrm{E}$ are the amplitudes of the two modelled cycles, $\mathrm{C}$ and $\mathrm{F}$ are the cycle duration in years, while $\mathrm{D}$ ang $\mathrm{G}$ account for the cosine phase shift. We fit all dv/v time series using a non-linear least squares approach. From the inversion, we find that for all selected time lapses the best-fit durations of the cycles are $\mathrm{C}=1 \pm 0.02 \mathrm{yr}$ and $\mathrm{F}=5 \pm 0.1 \mathrm{yrs}$.

The results of our fits are shown in figure 4 (red and green lines) together with the original data (blue lines). It can be observed that while the $\mathrm{dv} / \mathrm{v}$ amplitude of seasonal 1-yr oscillation (B) is higher at earlier lapse times, progressive decreases in the later coda are observed where $\mathrm{dv} / \mathrm{v}$ is more sensitive to the $5 \mathrm{yr}$ cycle $(\mathrm{E})$. To better 
visualize the results, we plot in figure 5 the $\mathrm{B}$ and $\mathrm{E}$ terms of equation 2 as function of coda lapse time. While the amplitude of the short-term cycle decreases at larger lapse time, the opposite behavior is observed for the amplitude of the 5-yrs (E). The ratio of the B and E parameters (fig. 5b) suggests that beyond 20s lapse time $\mathrm{dv} / \mathrm{v}$ is dominated by the long-term cycle.


Figure 4: $d v / v$ (blue) and modeled cycles with eq. 2 (red) for coda windows reported in table 1. The green line represents the long-term portion (first and third righthand terms of eq. 1). Numbers on top of each plot are the correlation coefficient between the model (eq. 2, red) and the data (blue).

The decay of the parameter B (fig. 3a) and the B/E ratio (fig. 3b), suggests that the source of the $1 \mathrm{yr}$ periodicity in $\mathrm{dv} / \mathrm{v}$ is dominantly at shallow depths (Obermann et al., 2013, see also the kernels in fig. 2). It has been suggested that thermally induced stresses rapidly decays with depth (Ben-Zion \& Leary, 1986), and the effects on dv/v have been reported in other regions (e.g. Richter et al, 2014). Thus, as the short-term cycle closely follows the seasonal temperature variation (fig. 3d, see fig S1) and shows a rapid decay as a function of depth, we infer that temperature plays a primary role in controlling the $\mathrm{dv} / \mathrm{v}$. 

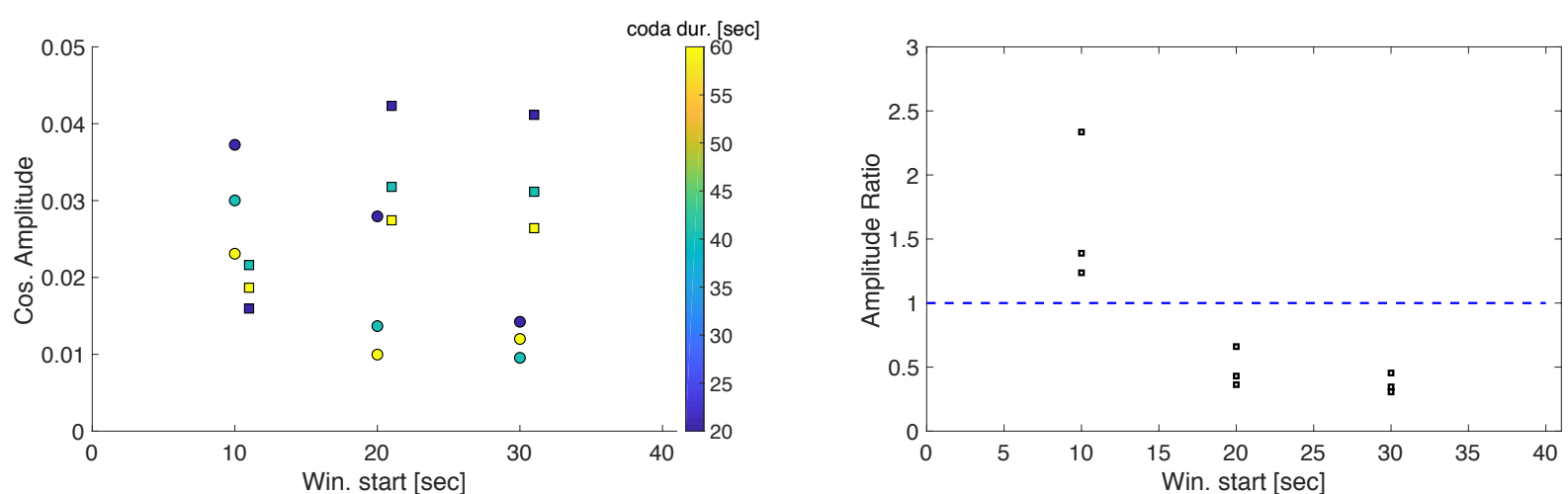

Figure 5: Magnitude of $B$ (round) and $E$ (squares) as function of coda window time (left). The color indicates the duration of the window. Squares are offset by 1sec for clarity. Ratio B/E as function of coda window order (right, black).

The long period oscillations of $\mathrm{dv} / \mathrm{v}$ are strongly correlated with the long-term variation of rainfall (fig. 3c) and GPS deformation (fig. 3e, S2). This multiyear signal represents transient deformations, occurring in highly fractured crustal rock as a response to variations of the hydraulic head in karst aquifers, and induces a strain rate up to $\sim 90$ nanostrain/yr (Silverii et al., 2019). This strain rate temporarily increases during high recharge, or diminishes during droughts, and can locally exceed the longterm secular tectonic strain rate (D'Agostino, 2014). The dv/v for later lapse times better correlates with this long period deformation (fig. 5). This observation suggests that a particular depth of the crust is more susceptible to these periodic perturbations (Obermann et al., 2013). Furthermore, we observe how for these late lapse-times (or depths) the short-term cycle has limited effect ( 2 to 4 times smaller, Fig. 5). As the thermally induced stress rapidly decays with depth (Ben-Zion \& Leary, 1986), we can further confirm that the late coda evolution of $\mathrm{dv} / \mathrm{v}$ is sensitive to deeper layers.

\section{b. Probing the crust with long term (5yrs) periodic deformations}

We probe the rheological response of the crust to these perturbations using time lapse measures of $\mathrm{dv} / \mathrm{v}$, similar to what has been done in other regions with tidal strain (Takano et al., 2014, Hillers et al., 2015b). To reiterate, the goal is to derive a lapse time (or depth) dependent measurements of $\mathrm{dv} / \mathrm{v}$ representative of perturbations at different crustal levels in order to reveal physical properties of rocks at different depth (e.g. Obermann et al., 2015, Hillers et al., 2018).

The in-situ measurements resemble laboratory dynamic acoustoelastic testing, a 'pump-probe' method (e.g., Renaud et al., 2009, 2012). Here, the medium changes are evaluated by estimating $\mathrm{dv} / \mathrm{v}$ using the reconstructed Green's function (the 'probe'), while the medium is deformed by long period ( $~ 5 \mathrm{yrs})$ perturbations (the 'pump') shown by the GPS signal (Silverii et al., 2019, Fig. 2). More precisely, we create a new set of autocorrelations by stacking 300 days of data with 200 days overlap. This time windowing helps us to reduce the short-term fluctuations (1yr). For each time segment $\mathrm{dv} / \mathrm{v}$ is estimated by comparing the estimated Green's function, with a reference signal from stacking over the full period (2000 to end of 2008). We use the stretching method and the same coda time windows listed in table 1 . The deformation is obtained from the projection of the east and north components of the GPS time series recorded at the AQUI station (Fig. 1), along the N45 direction, to maximize the amplitude of the hydrologically-related deformation ( $\widehat{\mathrm{d}}$ ) (Silverii et al., 2019). The GPS time series is indicative for the strain in the crust (Silverii et al., 2019): negative (south-westward) $\widehat{d}$ 
correspond to horizontal contraction, whereas positive $\widehat{\mathrm{d}}$ (north-eastward) values are representative of horizontal dilatation.

We then infer the sensitivity of $\mathrm{dv} / \mathrm{v}$ to deformation in each time window by fitting the data (fig. 6) with a linear model:

$$
\frac{\delta \mathrm{v}}{\mathrm{v}}=\alpha+\beta \hat{\mathrm{d}}[3]
$$

Equation 3 is similar to strain-dv/v relationship used to study the sensitivity to tidal strain (Takano et al., 2014, Hillers et al., 2015b), where $\beta$ represents the sensitivity to deformation while the zero-offset is $\alpha$. Our phenomenological model can be compared to the 1-D non-linear elasticity equation derived in Landau and Lifshutz (2012) and broadly applied to Earth materials (e.g., Guyer and Johnson, 1999;2009). We can interpret the sensitivity to elastic deformation $(\beta)$ as the classic non-linear elastic parameter that describes the slope of $\delta \mathrm{v} / \mathrm{v}$ over a single low frequency pump cycle. $\alpha$ is the non-linear volumetric change (length change in 1D) related to material conditioning (Guyer \& Johnson, 2009). Note in eq. 3 we do not include the cubic nonlinear elastic parameter that describes curvature in $\delta \mathrm{v} / \mathrm{v}$ as the scattering in the data provide similar misfit for $1^{\text {st }}$ and $2^{\text {nd }}$ order polynomial fit, nor the hysteretic term that is common to Earth materials.

The results in figure 6 show that, in agreement with experimental observations in laboratory rock samples (e.g. Renaud et al., 2012), velocity increases under compression (negative $\widehat{\mathrm{d}}$ ) and reduces during expansion episodes (positive $\widehat{\mathrm{d}}$ ). Similar results have been obtained by analyzing the relation between $\mathrm{dv} / \mathrm{v}$ and tidal strain using active source data (Yamamura et al., 2003) or noise correlation (Takano et al., 2014). A similar response to crustal dilatation has also been observed during tectonic transient deformation (Rivet et al., 2011). We also note that the parameter $\beta$ decreases with increasing lapse time (fig. 6). This lapse time evolution, combined with the depth kernels (fig. 2), suggests that the long-period strain perturbations sample primarily at depth (Obermann et al., 2015), rather than close to the free surface.

Before entering in the interpretation of this result, we will estimate the sensitivity to co-seismic strain perturbation (sec. 4). 

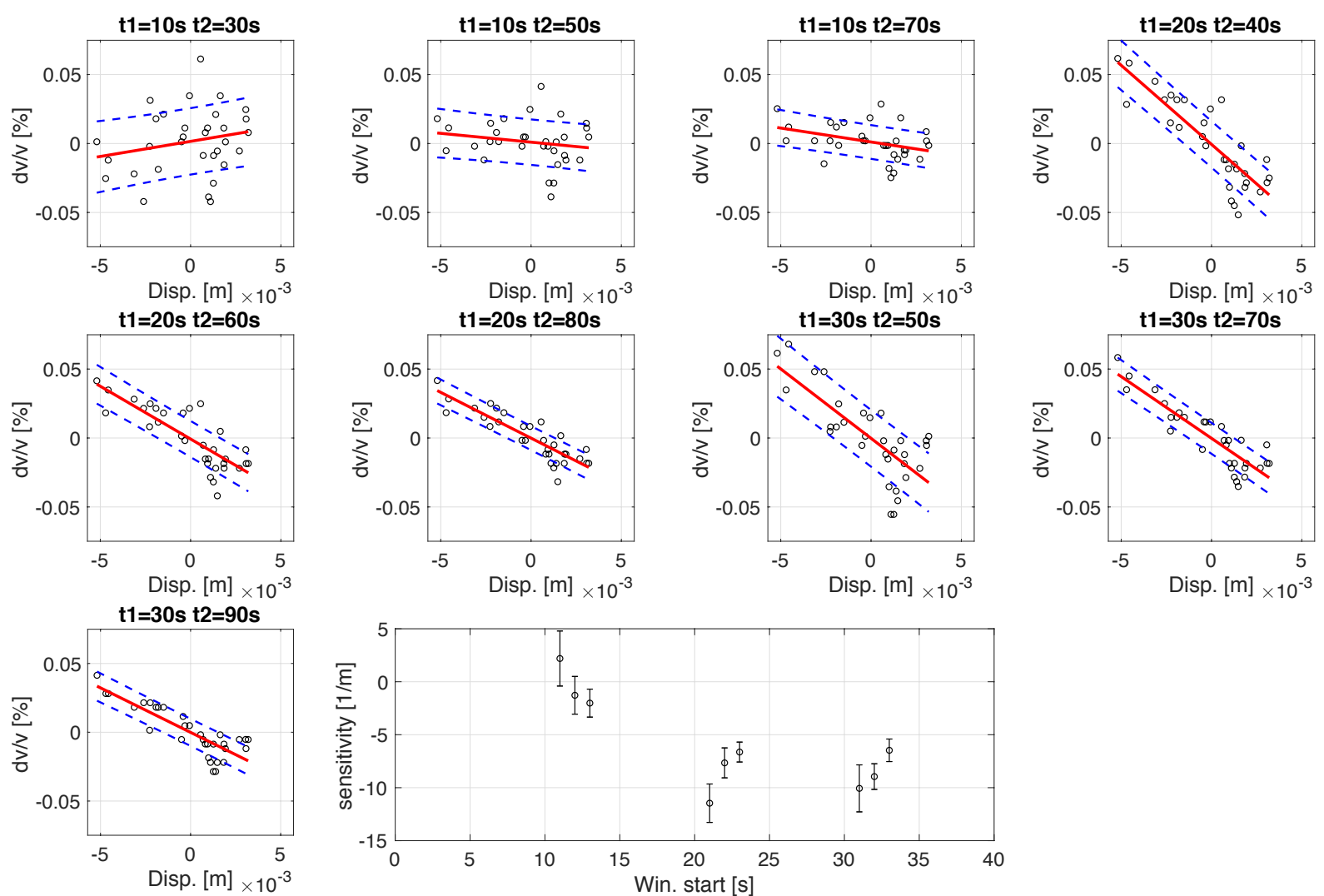

Figure 6: The scatter plot represents the fit of displacement and $d v / v$ using

equation 3. The last plot is the parameter $\beta$ of equation 3 as function of coda lapse time (Table 1).

\section{Estimation of sensitivity to co-seismic deformation}

The study region experienced three $M>6$ earthquakes during the period analyzed (2000-2017). The first is a magnitude Mw6.1 (Scognamiglio et al., 2010) occurring in 2009 near the city of L'Aquila with hypocentral depth at $8.3 \mathrm{~km}$. The station used to estimate the $\mathrm{dv} / \mathrm{v}$ is located close $(\sim 5 \mathrm{~km})$ to the epicenter and experienced a peak ground velocity of $35.8 \mathrm{~cm} / \mathrm{s}$ (esm.mi.ingv.it). In 2016 a series of moderate-large earthquakes (Norcia-Visso sequence) struck a region $\sim 40-70 \mathrm{~km}$ to the north of L'Aquila. The series began on the 24 of August 2016 with a magnitude 6 followed on the $30^{\text {th }}$ October 2016 by a magnitude 6.5 occurring in the same region. The last two events induced a peak ground velocity $\sim 9 \mathrm{~cm} / \mathrm{s}$ (esm.mi.ingv.it). The peak ground velocity is used to calculate the dynamic strain for the mentioned earthquakes (Taira et al, 2018, assuming $\mathrm{Vs}=2500 \mathrm{~m} / \mathrm{s}$ ), which is respectively $1.5 \mathrm{e}-4$ for L'Aquila event and $4 \mathrm{e}-5$ for the $24^{\text {th }}$ of August event in 2016.

In concomitance with the earthquakes, large velocity drops can be observed over the full range of the analyzed lapse times (fig. 7), similar to previous observations (Zaccarelli et al., 2011, Soldati et al., 2015). Nevertheless, we here measure the values of $\mathrm{dv} / \mathrm{v}$ for each time lapse and each earthquake, and we are thus able to resolve any depth dependence response of the crust to rapid dynamic perturbation induced by large earthquakes. For each event, the velocity reduction is measured as the drop from the mean $\mathrm{dv} / \mathrm{v}$ over the preceding year before the events, and the following minimum peak. For the 2016 events, our analysis window (90 days) prevents us from resolving the two drops (for the 24 of August and the 30 October events respectively). We thus consider the mean reference $d v / v$ for the year before the first event ( $24^{\text {th }}$ of August 2016). 
The co-seismic $\mathrm{dv} / \mathrm{v}$ at different lapse time (Fig. 7) shows a different evolution with respect to multiyear ones (Fig. 5 and 7). For both events, we see a general reduction of $\mathrm{dv} / \mathrm{v}$ as time lapse increases.
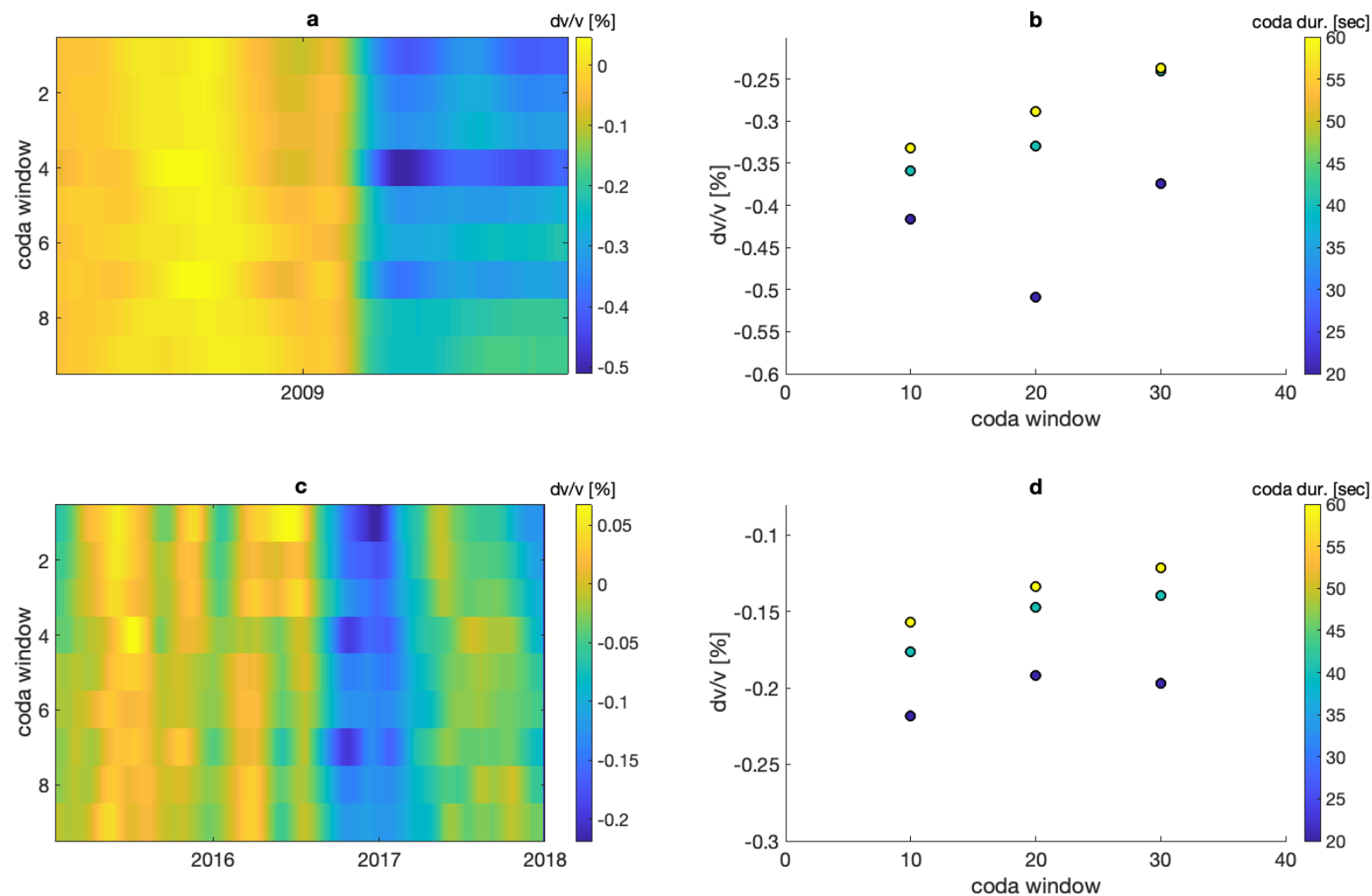

Figure 7: Coseismic dv/v time series for different lapse time (Table 1) for L'Aquila 2009 event (a) and Visso-Norcia sequence 2016 (c). Coseismic velocity reduction as function of coda lapse time (Table 1) for L'Aquila 2009 event (b) and Visso-Norcia sequence 2016 (d).

\section{Discussion}

The analysis of the 17 years of $\mathrm{dv} / \mathrm{v}$ permitted us to isolate several processes controlling $\mathrm{dv} / \mathrm{v}$ variations. Namely we observe velocity change due to (i) co-seismic perturbations (Fig. 7), (ii) yearly perturbations likely related to variation of temperature (Fig. 4, 5) and (iii) multiannual perturbations associated with hydrological cycles inducing dilatational strain in the crust (Fig. 4, 5, 6, Silverii et el., 2019). By estimating dv/v over different coda lapse time (Table 1 ), we observe how the contribution from each process changes over different portions of the correlation coda (see Table 2, Fig. 5, 6, 7).

\section{a. Depth resolution of velocity variations}

To interpret the results for different lapse times in term of depth, we calculated the sensitivity kernel based on the single scattering 3D radiative transfer solution considering that the measured coda waves are comprised of contributions from both body and surface waves. It is important to note that, as we have no proxy to assess the coda wave constituents, this kernel can be biased. The sensitivity to surface waves is more important for early lapse times (Obermann et al., 2013). Here we assume the value of the mean free path $(1=100 \mathrm{~km}$ and 10 $\mathrm{km}$ ) and propagation velocity of coda waves $\sim 3895 \mathrm{~m} / \mathrm{s}$, for the equipartition state. Thus, the inferred depth sensitivity is not absolute, but we can use the kernels as an indicator of the approximate depth with lapse time. The estimated sensitivity (Fig. 2) maximum is 
approximately $\sim 10 \mathrm{~km}$ especially for the later coda (e.g. lapse time 20 - 50s) with $50 \mathrm{~km}$ scattering mean free path.

The estimation of an absolute depth for the velocity changes will require better knowledge of the scattering properties of the medium, including its layered structure, which can play a fundamental role in trapping waves in some part of the medium (e.g. Kanu \& Snieder, 2015). Here, we can only discuss the relative depth of the velocity changes observed (multiyear, seasonal, co-seismic, Fig. 5, 7) from the time lapse evolution of dv/v. For example, for multiyear deformations dv/v increases for later lapse-time (Fig. 5, 7). We thus infer the existence of a region at depth that exhibits sensitivity to long period forcing (Obermann et al., 2013, Hillers et al., 2018). The rapid decay of the B/E ratio (eq. 2, Fig. 5b), is another indication that our late coda measurements sample deeper into the crust. In fact, the thermally induced stress is expected to reduce rapidly with depth (Ben-Zion \& Leary, 1986) as does the ratio (Fig. 5b).

\section{b. Origin of various velocity variation and rheology of middle crust}

We begin by discussing the strain sensitivity $(e=[d v / v] /[d e]$, de the dynamic strain) during the co-seismic stage. Considering the dynamic strain induced by the two events we find that $e$ is respectively $-1 \mathrm{e} 7$ and $-1 \mathrm{e} 8$ for the L'Aquila and Amatrice earthquakes respectively. These values are an order of magnitude larger than estimates in geothermal areas (Taira et al., 2018) and volcanic regions (Brenguier et al., 2014), suggesting that the crustal rocks in the study region are very susceptible to dynamic deformations. Under rapid perturbations induced during large earthquakes the $\mathrm{dv} / \mathrm{v}$ decreases with depth, suggesting significant nonlinear response related to damage near the surface (e.g. Obermann, 2013, 2014), or alternatively, unconsolidated granular material (e.g., Brunet et al., 2008; Johnson and Jia, 2005). For the 2009 event, considering the shortest analysis window in the coda (20s, Fig. 7) a peak of maximum velocity reduction is observed, which suggests the existence of an isolated region at depth that is highly sensitive to dynamic deformations. The detailed analysis of the co-seismic response is beyond the scope of the actual work, and will be addressed in future research.

While the co-seismic rapid shaking induces large $\mathrm{dv} / \mathrm{v}$ near the surface, the $\mathrm{dv} / \mathrm{v}$ increases with lapse time for the long-term perturbations (Table 2, Figure 5, 6). We interpret this time lapse evolution as due to an isolated region with stronger $\mathrm{dv} / \mathrm{v}$-sensitivity to perturbations (sec. a, Obermann et al., 2013, 2014, Hillers et al., 2018). While we do not have detailed evolution of strain for the long-term dv/v, we can make a back-of-the-envelope calculation of the sensitivity, knowing that the strain at peak GPS deformation is $\sim 1 \mathrm{e}-6$ (Silverii et al., 2019) for $\sim 1 \mathrm{e}-3$ of $\mathrm{dv} / \mathrm{v}$, which gives a dv/v-sensitivity of $\sim 1 \mathrm{e}$. This latter value agrees with other estimates based on tidal-dv/v responses (Yamamura et al., 2003, Takano et al. 2014). Our estimations are also in agreement with dilatant induced velocity reduction during slow slip in the lower crust (Rivet et al., 2011, Wang et al., 2019).

Laboratory studies show that nonlinear elastic modulus variation is amplitude dependent (Guyer and Johnson, 2009) as well as frequency dependent, with larger modulus changes for shorter periods for a given effective pressure (Riviere et al., 2016). Here we observe a lower $\mathrm{dv} / \mathrm{v}$ sensitivity (1e3) for small quasi-static strain (1e-6) and low frequency ( $\sim 5 \mathrm{yrs)}$ in contrast to large dynamic strains $(\sim 1 \mathrm{e}-4)$ (lasting no more than few minutes) induced by the large earthquakes. Under low strain forcing we also observe that non-linear material slow dynamics (recovery) expressed in equation 3 is nearly zero ( $\alpha$, Table 2$)$. However, for short time perturbations a clear log-time recovery is observed (see for example the time after the earthquakes in figure 3a). Thus, our measures reflect general behaviors of rocks in small scale laboratory experiments (e.g. Riviere et al., 2016) as well as field measurements under Vibroseis forcing (Johnson et al., 2008) and earthquake forcing (e.g., Brengieur et al., 2008; Wu et al., 2017; Ostrovsky et al, 2019). 
To interpret our results, we consider the velocity variation as being controlled by crack density $\left(\rho_{0}\right)$ because it has been well documented that cracks and other damage contribute dominantly to nonlinear elastic behavior (e.g., Johnson 1998; Guyer and Johnson, 2009). The sensitivity to any stress perturbations (S) can then be written as (Silver et al., 2007):

$$
\mathrm{e}=\frac{\delta \mathrm{v} / \mathrm{v}}{\mathrm{s}}=\frac{\delta \mathrm{v} / \mathrm{v}}{\Delta \rho_{0}} \frac{\Delta \rho_{0}}{\mathrm{~s}}[4]
$$

As the crack density is expected to reduce with depth due to the lithostatic pressure (Tod, 2003), sensitivity should reduce for larger lapse time (or depth) assuming fluid pressures do not vary radically. For a fixed depth dependence of strain, at shallow depth we expect larger sensitivity for larger strains, as observed during the co-seismic shaking. We again note how our results are anomalous for long-term deformation, as the sensitivity increases at depth (Table 2, Figure 5,6). The observed dichotomy (shallow response during co-seismic forcing and deeper response during long-term forcing) is similar to what has been observed in Wenchuan area by Obermann et al., (2014).

The cause of velocity variation at late lapse time may be due to meteorological water circulation in the crust. However, in this case we would expect velocity changes also at shallow depth (early lapse time). Thus, alternative mechanism(s) inducing changes may exist. A possibility is that forcing induced by water table variation, rather than water itself, is responsible for velocity changes, with the loading (unloading) inducing significantly increment (reduction) of the pore pressure and controlling the drop (increment) of velocity (Froment et al., 2013, Obermann et al., 2014). This mechanism has also been observed and modeled in the Irpinia region (D'Agostino et al., 2018).

The presence of pressurized fluids at depth a range $5-10 \mathrm{~km}$ has been suggested by several researchers for the region of L'Aquila, from analysis of earthquake source properties (Tarekawa et al., 2010, Malagnini et al., 2012) or from Vp/Vs ratio (Di Luccio et al, 2012). Other studies suggest an extensive dilatancy anisotropy in the central Apennines, related to pervasive fluid-filled cracks oriented parallel to the Apennines chain (Pastori et al., 2016). Furthermore, a significant presence of a damage zone comprised of near-fault cracks (up to $\sim 1 \mathrm{~km}$ from the fault) are observed in the carbonate rocks near the region of L'Aquila (Agosta \& Aydin, 2006).

Putting together our results with the other geophysical information, suggests the existence of a depth-isolated and long-lived (at least 9 years), intensely cracked and fluid rich, damaged region in the study area. At this depth, the strong sensitivity (equation 3 and 4) is related to the presence of fluids, reducing the effective pressure, thus favoring high crack density (Hamiel et al., 2006) and making the material more susceptible to dynamic or quasi-static forcing.

Mid-crustal damage zones have been also observed in California by analysis of seismic catalogs (Ben-Zion \& Zaliapin, 2019). Highlighting the existence of such a damage zone is fundamental as damage plays a key role in the evolution of the seismic cycle and earthquake nucleation (Lyakhovsky et al., 2001, Renard et al., 2018) and controls the amount of strain released in a brittle manner during the seismic cycle (Hamiel et al., 2006). For example, numerical simulations show that nucleation and growth of large earthquakes is only possible with the existence of a, at least modestly, damaged region (Lyakhovsky et al., 2001). Indeed, Ben-Zion \& Zaliapin (2019) highlighted significant damage volumes active prior to several significant earthquakes in California. In a similar manner, intense seismic activity was observed, concentrated near the nucleation depth of L'Aquila earthquake, in the last year preceding the mainshock (e.g. Valoroso et al., 2013). Whereas seismicity solely provides a volume of damage (Ben-Zion \& Zaliapin, 2019), we quantitatively estimate rheological parameters (e.g. sensitivity) of rocks at depth in a region of important seismic hazard. 
The presence of a highly fractured rocks has implication for earthquake nucleation models. others (Renard et al, 2018). In this model, cracks become more interconnected as a large event is approaching, and progressively merge into a single primary fracture (the main shock). Close to the main rupture significant precursory earthquakes must occur in the damage zone (Renard et al., 2018), similarly to what is observed in the year preceding the L'Aquila earthquake in 2009 (e.g. Valoroso et al., 2013).

We finally note (fig. 3a) how the seasonal cycle seems to disappear after the 2009 L'Aquila earthquake. Given that rain cycle remains significant after 2009, this behavior seems to suggest less sensitivity of the medium after the earthquake. However, there might exists a possible interplay between the long and slow post-seismic recovery and multiyear cycle, which will be studied in future works. Further, fluid pressures could play a key role-decreased pressures allow cracks to close and elastic non-linearity to decrease. This point needs further work.

Table 2: Summary of derived parameters

\begin{tabular}{|c|c|c|c|c|c|c|}
\hline $\begin{array}{l}\text { Window } \\
\text { number }\end{array}$ & $\begin{array}{c}\text { 1-year cycle } \\
\text { (eq. 2) }\end{array}$ & $\begin{array}{c}5 \text { years } \\
\text { cycle (eq. } \\
\text { 2) }\end{array}$ & $\alpha(e q .3)$ & $\begin{array}{c}\boldsymbol{\beta}(e q . \\
3)\end{array}$ & $\begin{array}{c}L ' \text { Aquila } d v / v \\
P G V=35.8 \mathrm{~cm} / \mathrm{s} \\
\text { Strain }=1.5 \mathrm{e}-4\end{array}$ & $\begin{array}{c}\text { Amatrice } \\
d v / v \\
P G V=9 \text { eqp } 1 S \\
\text { Strain }=4 e-5\end{array}$ \\
\hline 1 & 0.036 & 0.01 & 0.006 & 1.65 & -0.39 & -0.18 \\
\hline 2 & 0.02 & 0.02 & 0.005 & -1.22 & -0.34 & -0.1543 \\
\hline 3 & 0.02 & 0.01 & 0.004 & -2.22 & -0.31 & -0.12 \\
\hline 4 & 0.02 & 0.04 & 0.008 & -11.56 & -0.49 & -0.1545 \\
\hline 5 & 0.01 & 0.03 & 0.005 & -7.83 & -0.32 & -0.11 \\
\hline 6 & 0.00 & 0.02 & 0.004 & -6.82 & -0.28 & טדם \\
\hline 7 & 0.01 & 0.04 & 0.008 & -8.79 & -0.36 & -0.15 \\
\hline 8 & 0.00 & 0.03 & 0.005 & -8.76 & -0.23 & $-0.1 \bar{\theta}^{48}$ \\
\hline 9 & 0.00 & 0.02 & 0.004 & -6.36 & -0.23 & -0.08 \\
\hline
\end{tabular}

\section{a. Towards monitoring with seasonal loading}

We showed that seasonal and multiyear components dominate the $\mathrm{dv} / \mathrm{v}$ time series in the inter-seismic period (figs. 4, 5). These cycles are likely to mask smaller tectonic signals (e.g. fault weakening during earthquake preparation or transient tectonic deformations among). Indeed, systematic efforts have been applied to characterize the cyclic variations by modeling them (Wang et al. 2017) or by estimating the repeating patterns over several cycles and use their average as correction to isolate tectonic signals (Hillers et al., 2018).

Knowing the physical processes responsible for multiannual dv/v evolution (Silverii et al, 2019), we assessed the lapse time dependent response of the medium to these cycles of dilatational strain, thus turning a nuisance into an opportunity to study the crust in a region of significant seismic hazard. This approach is similar to the studies of tidal induced dv/v (Takano et al., 2014, Hillers et al., 2015b), but we here assess longer cycles, and sample deeper regions in the crust near a main active fault.

Our lapse time $\mathrm{dv} / \mathrm{v}$ analysis bears similarities to laboratory dynamic nonlinear studies applied to resolve the spatial extent of damaged regions in solids (e.g., Johnson, 1998; Ulrich et al., 2007; Guyer and Johnson, 2009; Ostrovsky and Johnson, 2001; Haupert et al., 2014). As in these studies we observe a maximum response to deformation of the medium ( $\beta$, eq. 3 ) in the damaged zone. Strain concentration can also produce highly localized increases in nonlinear response (Lott et al., 2016) especially at crack tips (e.g., Ulrich 2006), and thus time lapse monitoring could be used to reveal zones of anomalous strain accumulation.

The extension of our analysis to other regions, instrumented with dense geodetic networks and or application of InSAR allowing the accurate estimate of time-dependent strain, will help to better assess the physical properties of rocks at depth (e.g. assessing conditioning, slow 
dynamics and hysteresis [Guyer and Johnson, 2009; TenCate et al., 2000]). Potential regions for new studies include New Madrid seismic zone (Craig et al., 2017) or the Himalayan region (Bettinelli et al., 2008, Bollinger et al., 2007), or Irpinia (D’Agostino et al., 2018). These regions are characterized by significant seismic risk, prominent seasonal and multiannual perturbations, and excellent seismological and geodetic instrumentation.

\section{Conclusions}

The precise analysis of time lapse velocity variations for 17 years of data in the region of L'Aquila permitted us to unravel the complex behavior of multiple processes controlling the $\mathrm{dv} / \mathrm{v}$ evolution, over a wide range of temporal and spatial scales. The comparison of $\mathrm{dv} / \mathrm{v}$ with independent measurements (e.g. dynamic strain induced by earthquakes, quasi-static strain due to hydrological cycles) permitted us to characterize the rheological response of seismogenic rocks to various level of strain at various depths. The time-lapse analysis allowed us to resolve a dichotomy in the crustal response, with significant near surface damage due to rapid strain induced by large earthquakes and deeper strain sensitivity due to long period (5yrs) and small strain perturbations in an inferred damage zone containing high fluid pressures, where the mainshock earthquake nucleates.

We showed that when the physical processes responsible for seasonal or multiyear cycles can be quantitatively characterized (e.g. Silverii et al., 2019), they can be exploited to evaluate the rheological properties of rocks in the crust. This work extends previous approaches based on tidal strain-dv/v evaluation, which are primarily sensitive to the shallowest part of the crust $(<\sim 1 \mathrm{~km}$, e.g. Takano et al. 2014). Furthermore, our analysis made it possible to turn hydrologically-induced cycles of $\mathrm{dv} / \mathrm{v}$, usually seen as a nuisance as they can mask tectonic events, into an opportunity to reveal the rheology of crustal rocks down to the seismogenic depth. Extending this work to other seismic active region, affected by significant weatherrelated cycles (Bettinelli et al., 2008, Bollinger et al., 2007, D’Agostino et al., 2018, Craig et al., 2017) will reveal new information about the physical properties of near fault rocks.

\section{Acknowledgements}

This research received funding from the European Research Council (ERC) under the European Union Horizon 2020 Research and Innovation Programme (grant agreements, 802777-MONIFAULTS). PAJ was supported by the US DOE Office of Science, Chemical Sciences, Geosciences, and Biosciences. QW was supported by the ERCproject 742335-FIMAGE. The article benefited greatly from discussions with Yehuda Ben-Zion, William Frank, Michele Fondriest, Ivan Callegari, Michel Campillo, Martin Lott and Leonard Seydoux. The rain and temperature data have were graciously provided by the Ufficio Idrografico Mareografico of the Abruzzo region. The seismic data are available at Instituto Nazionale di Geofisica e Vulcanologia (http://iside.rm.ingv.it/instruments) and at Incorporated Research Institution for Seismology (https://www.iris.edu/hq/).

\section{Bibliography}

Agosta, Fabrizio, and Atilla Aydin. "Architecture and deformation mechanism of a basinbounding normal fault in Mesozoic platform carbonates, central Italy." Journal of Structural Geology 28.8 (2006): 1445-1467.

Avallone, Antonio, et al. "The RING network: improvement of a GPS velocity field in the central Mediterranean." Annals of Geophysics 53.2 (2010): 39-54. 
Ben-Zion, Yehuda. "Collective behavior of earthquakes and faults: Continuum-discrete transitions, progressive evolutionary changes, and different dynamic regimes." Reviews of Geophysics 46.4 (2008).

628

Ben-Zion, Yehuda, and Peter Leary. "Thermoelastic strain in a half-space covered by unconsolidated material." Bulletin of the Seismological Society of America 76.5 (1986): 14476311460.

632

Ben-Zion, Yehuda, and Ilya Zaliapin. "Spatial variations of rock damage production by earthquakes in southern California." Earth and Planetary Science Letters 512 (2019): 184193.

Bettinelli, Pierre, et al. "Seasonal variations of seismicity and geodetic strain in the Himalaya induced by surface hydrology." Earth and Planetary Science Letters 266.3-4 (2008): 332-344.

Bollinger, L., et al. "Seasonal modulation of seismicity in the Himalaya of Nepal." Geophysical Research Letters 34.8 (2007).

Brenguier, F., et al. "Mapping pressurized volcanic fluids from induced crustal seismic velocity drops." Science 345.6192 (2014): 80-82.

Brenguier, Florent, et al. "Postseismic relaxation along the San Andreas fault at Parkfield from continuous seismological observations." science 321.5895 (2008): 1478-1481.

Brunet, T., X. Jia and P. Johnson, Transitional, elastic-nonlinear behaviour in dense granular media, Geophys. Res. Lett. 35, L19308-L19311 doi:10.1029/2008GL035264 (2008).

Chiaraluce, L., et al. "The 2009 L'Aquila (Central Italy) Seismic Sequence." Bollettino di Geofisica Teorica e Applicata. (2010).

655

656

657

658

659

660

661

662

Chiaraluce, L., et al. "The anatomy of the 2009 L'Aquila normal fault system (central Italy) imaged by high resolution foreshock and aftershock locations. "Journal of Geophysical Research: Solid Earth 116.B12 (2011).

Craig, Timothy J., Kristel Chanard, and Eric Calais. "Hydrologically-driven crustal stresses and seismicity in the New Madrid Seismic Zone." Nature communications 8.1 (2017): 2143.

Chiaraluce, Lauro, et al. "The 2016 central Italy seismic sequence: A first look at the mainshocks, aftershocks, and source models." Seismological Research Letters 88.3 (2017): 757-771.

Chiaraluce, L. "Unravelling the complexity of Apenninic extensional fault systems: a review of the 2009 L'Aquila earthquake (Central Apennines, Italy)." Journal of Structural Geology 42 (2012): 2-18.

D'Agostino, N. "Complete seismic release of tectonic strain and earthquake recurrence in the 673 
D'Agostino, Nicola, et al. "Crustal deformation and seismicity modulated by groundwater recharge of karst aquifers." Geophysical Research Letters 45.22 (2018): 12-253.

Delorey, Andrew A., Nicholas J. van der Elst, and Paul A. Johnson. "Tidal triggering of earthquakes suggests poroelastic behavior on the San Andreas Fault." Earth and Planetary Science Letters 460 (2017): 164-170. Mw 6.3 L'Aquila earthquake, central Italy." Journal of Geophysical Research: Solid 683 Earth 115.B6 (2010).

684

685

686

687

688

689

690

691

Froment, B., et al. "Deformation at depth associated with the 12 May 2008 Mw 7.9 Wenchuan earthquake from seismic ambient noise monitoring." Geophysical Research Letters 40.1 (2013): 78-82.

Guyer, Robert A., and Paul A. Johnson. "Nonlinear mesoscopic elasticity: Evidence for a new class of materials." Physics today52 (1999): 30-36.

Guyer, Robert A., and Paul A. Johnson. Nonlinear mesoscopic elasticity: the complex behaviour of rocks, soil, concrete. John Wiley \& Sons, 2009.

Hamiel, Yariv, et al. "Stable and unstable damage evolution in rocks with implications to fracturing of granite." Geophysical Journal International 167.2 (2006): 1005-1016.

Herrmann, R. B. (2013). Computer programs in seismology: An evolving tool for instruction and research. Seismological Research Letters, 84(6), 1081-1088. https://doi.org/10.1785/0220110096

Haupert, Sylvain, et al. "Optimized dynamic acousto-elasticity applied to fatigue damage and stress corrosion cracking." Journal of Nondestructive Evaluation 33.2 (2014): 226-238.

Hillers, G., et al. "Seasonal variations of seismic velocities in the San Jacinto fault area observed with ambient seismic noise." Geophysical Journal International 202.2 (2015a): 920932.

Hillers, G., et al. "In situ observations of velocity changes in response to tidal deformation from analysis of the high-frequency ambient wavefield." Journal of Geophysical Research: Solid Earth120.1 (2015b): 210-225.

Hillers, Gregor, et al. "Transient change of seismic velocities in the San Jacinto fault region following the 2010 M7. 2 El Mayor-Cucapah earthquake observed with ambient noise monitoring." EGU General Assembly Conference Abstracts. Vol. 20. 2018.

Johnson, P. A. and X. Jia, Nonlinear dynamics, granular media and dynamic earthquake triggering, Nature, 473 871-874 (2005)

Johnson, P., Robert Guyer, and L. Ostrovsky. A nonlinear mesoscopic elastic class of materials. No. LA-UR-99-4733. Los Alamos National Lab., NM(US), 1999. 
Johnson, Paul A. "The new wave in acoustic testing." Materials World (1999): 544-546.

Johnson, Paul A., et al. "Inducing in situ, nonlinear soil response applying an active source." Journal of Geophysical Research: Solid Earth 114.B5 (2009).

Kanu, Chinaemerem, and Roel Snieder. "Numerical computation of the sensitivity kernel for monitoring weak changes with multiply scattered acoustic waves." Geophysical Supplements to the Monthly Notices of the Royal Astronomical Society 203.3 (2015): 1923-1936.

Lacombe, Céline, et al. "Separation of intrinsic absorption and scattering attenuation from Lg coda decay in central France using acoustic radiative transfer theory." Geophysical Journal International 154.2 (2003): 417-425.

\section{L.D. Landau \& E.M. Lifshitz Theory of Elasticity (Volume 7 of A Course of Theoretical} Physics) Pergamon Press 1970

Lobkis, O. I., and R. L. Weaver (2003), Coda-wave interferometry in finite solids: Recovery of P-to-S conversion rates in an elastodynamic billiard, Phys. Rev. Lett., 90(25), 254302, doi:10.1103/PhysRevLett.90.254302.

Lott, Martin, et al. "From local to global measurements of nonclassical nonlinear elastic effects in geomaterials." The Journal of the Acoustical Society of America 140.3 (2016): EL231-EL235.

Lott, Martin, et al. "Three-dimensional modeling and numerical predictions of multimodal nonlinear behavior in damaged concrete blocks." The Journal of the Acoustical Society of America 144.3 (2018): 1154-1159.

Lyakhovsky, V., Ben-Zion, Y., Agnon, A., 2001. Earthquake cycle, fault zones, and seismicity patterns in a rheologically layered lithosphere. J. Geophys. Res. 106, 4103-4120.

Malagnini, Luca, et al. "Control of pore fluid pressure diffusion on fault failure mode: Insights from the 2009 L'Aquila seismic sequence." Journal of Geophysical Research: Solid Earth 117.B5 (2012).

Margerin, Ludovic, Michel Campillo, and Bart Van Tiggelen. "Monte Carlo simulation of multiple scattering of elastic waves." Journal of Geophysical Research: Solid Earth 105.B4 (2000): 7873-7892.

McCall, K. R., and R. A. Guyer. Hysteresis and nonlinear elasticity in rocks. No. LA-UR-934143; CONF-9308167-5. Los Alamos National Lab., NM (United States), 1993.

Obermann, Anne, et al. "Depth sensitivity of seismic coda waves to velocity perturbations in an elastic heterogeneous medium." Geophysical Journal International 194.1 (2013): 372-382.

Obermann, Anne, et al. "Seismic noise correlations to image structural and mechanical changes associated with the Mw 7.92008 Wenchuan earthquake." Journal of Geophysical Research: Solid Earth 119.4 (2014): 3155-3168. 
Ostrovsky, L. A., and P. A. Johnson. "Dynamic nonlinear elasticity in geo materials." Rivista del Nuovo Cimento della Societa Italiana di Fisica 24.7 (2001): 1-46.

Ostrovsky, Lev, et al. "Long-Time Relaxation Induced by Dynamic Forcing in Geomaterials." Journal of Geophysical Research: Solid Earth (2019).

Pacheco, Carlos, and Roel Snieder. "Time-lapse travel time change of multiply scattered acoustic waves." The Journal of the Acoustical Society of America 118.3 (2005): 1300-1310.

Paasschens, J. C. J. "Solution of the time-dependent Boltzmann equation." Physical Review E 56.1 (1997): 1135.

Pastori, Marina, Paola Baccheschi, and Lucia Margheriti. "Shear wave splitting evidence and relations with stress field and major faults from the "Amatrice-Visso-Norcia Seismic Sequence"." Tectonics (2019).

Planès, Thomas, et al. "Decorrelation and phase-shift of coda waves induced by local changes: multiple scattering approach and numerical validation." Waves in Random and Complex Media24.2 (2014): 99-125.

Poli, P., et al. "Noise directivity and group velocity tomography in a region with small velocity contrasts: the northern Baltic shield." Geophysical Journal International 192.1 (2012): 413 424.

Poupinet, G., W. Ellsworth, and J. Frechet (1984), Monitoring velocity variations in the crust using earthquake doublets: An application to the Calaveras Fault, California, J. Geophys. Res., 89(B7), 5719-5731.

Renard, F., Weiss, J., Mathiesen, J., Ben Zion, Y., Kandula, N., Cordonnier, B., 2018. Critical evolution of damage towards system-size failure in crystalline rock. J. Geophys. Res. 123, 1969-1986. https://doi.org/10.1002/2017JB014964.

Renaud, Guillaume, Samuel Callé, and Marielle Defontaine. "Remote dynamic acoustoelastic testing: Elastic and dissipative acoustic nonlinearities measured under hydrostatic tension and compression." Applied Physics Letters 94.1 (2009): 011905.

Renaud, G., P-Y. Le Bas, and P. A. Johnson. "Revealing highly complex elastic nonlinear (anelastic) behavior of Earth materials applying a new probe: Dynamic acoustoelastic testing." Journal of Geophysical Research: Solid Earth 117.B6 (2012).

Richter, Tom, et al. "Comprehensive observation and modeling of earthquake and temperature-related seismic velocity changes in northern Chile with passive image interferometry." Journal of Geophysical Research: Solid Earth 119.6 (2014): 4747-4765.

Rivet, Diane, et al. "Seismic evidence of nonlinear crustal deformation during a large slow slip event in Mexico." Geophysical Research Letters 38.8 (2011).

Rivière, J., L. Pimienta, M. Scuderi, T. Candela, P. Shokouhi, J. Fortin,A. Schubnel, C. Marone, and P. A. Johnson (2016), Frequency, pressure, and strain dependence of nonlinear elasticity in Berea Sandstone, Geophys. Res. Lett.,43, 3226-3236,doi:10.1002/2016GL068061. 
818 Rivière, Jacques \& Shokouhi, Parisa \& Guyer, Robert \& Johnson, Paul. (2015). A set of 819 measures for the systematic classification of the nonlinear elastic behavior of disparate rocks.

820

821

822

823

824

825

826

827

828

829

830

831

832

833

834

835

836

837

838

839

840

841

842

843

844

845

846

847

848

849

850

851

852

853

854

855

856

857

858

859

860

861

862

863

864

865

866

867

Journal of Geophysical Research: Solid Earth. 120. 10.1002/2014JB011718.

Sabra, Karim G., et al. "Extracting time-domain Green's function estimates from ambient seismic noise." Geophysical Research Letters 32.3 (2005).

Scognamiglio, L., Tinti, E., Quintiliani, M. (2006). Time Domain Moment Tensor [Data set]. Istituto Nazionale di Geofisica e Vulcanologia (INGV). https://doi.org/10.13127/TDMT

Scognamiglio, Laura, et al. "Fast determination of moment tensors and rupture history: What has been learned from the 6 April 2009 L'Aquila earthquake sequence." Seismological Research Letters 81.6 (2010): 892-906.

Sens-Schönfelder, Christoph, Roel Snieder, and Xun Li. "A model for nonlinear elasticity in rocks based on friction of internal interfaces and contact aging." Geophysical Journal International216.1 (2018): 319-331.

Soldati, Gaia, et al. "Monitoring of crustal seismic velocity variations in the L'Aquila fault zone inferred from noise cross-correlation." Geophysical Journal International 202.1 (2015): 604-611.

Shapiro, Nikolai M., and Michel Campillo. "Emergence of broadband Rayleigh waves from correlations of the ambient seismic noise." Geophysical Research Letters 31.7 (2004).

Silver, Paul G., et al. "Active source monitoring of cross-well seismic travel time for stressinduced changes." Bulletin of the Seismological Society of America 97.1B (2007): 281-293.

Silverii, Francesca, et al. "Transient deformation of karst aquifers due to seasonal and multiyear groundwater variations observed by GPS in southern Apennines (Italy). "Journal of Geophysical Research: Solid Earth 121.11 (2016): 8315-8337.

Silverii, Francesca, et al. "Transient crustal deformation from karst aquifers hydrology in the Apennines (Italy)." Earth and Planetary Science Letters 506 (2019): 23-37.

TenCate, J.A., E. Smith, and R.t A. Guyer, Universal Slow Dynamics in Granular Solids. Phys. Rev. Lett. 85, 1020 (2000)

Van Den Abeele, Koen \& Visscher, Joëlle. (2000). Damage Assessment in reinforced concrete using spectral and temporal nonlinear vibration techniques. Cement and Concrete Research. 30. 1453-1464. 10.1016/S0008-8846(00)00329-X.

van der Elst, Nicholas J., et al. "Fortnightly modulation of San Andreas tremor and lowfrequency earthquakes." Proceedings of the National Academy of Sciences 113.31 (2016): 8601-8605.

Valoroso, L., et al. "Radiography of a normal fault system by 64,000 high-precision earthquake locations: The 2009 L'Aquila (central Italy) case study." Journal of Geophysical Research: Solid Earth 118.3 (2013): 1156-1176. 
868 Weaver, Richard L. "On diffuse waves in solid media." The Journal of the Acoustical Society 869 of America 71.6 (1982): 1608-1609.

870

Yamamura, Keiko, et al. "Long-term observation of in situ seismic velocity and attenuation." Journal of Geophysical Research: Solid Earth 108.B6 (2003).

873

Taira, Taka'aki, et al. "Monitoring reservoir response to earthquakes and fluid extraction, Salton Sea geothermal field, California." Science Advances 4.1 (2018): e1701536.

876

Takano, Tomoya, et al. "Seismic velocity changes caused by the Earth tide: Ambient noise correlation analyses of small-array data." Geophysical Research Letters 41.17 (2014): 61316136.

Terakawa, Toshiko, et al. "High-pressure fluid at hypocentral depths in the L'Aquila region inferred from earthquake focal mechanisms." Geology 38.11 (2010): 995-998.

Tod, S. R. "Bed-limited cracks in effective medium theory." Geophysical Journal International 152.2 (2003): 344-352.

Ulrich, T. J., P. A. Johnson, and R. A. Guyer, Interaction Dynamics of Elastic Waves with a Complex Nonlinear Scatterer through the Use of a Time Reversal Mirror, Phys. Rev. Lett. 98,

889

890

891

892

893 104301 (2007).

Wang, Qing-Yu, et al. "Seasonal crustal seismic velocity changes throughout Japan." Journal of Geophysical Research: Solid Earth122.10 (2017): 7987-8002.

Wang, Qing-Yu, et al. "Evidence of changes of seismic properties in the entire crust beneath Japan after the Mw 9.0, 2011 Tohoku-oki earthquake." Journal of Geophysical Research: Solid Earth (2019).

$898 W$ W, Chunquan, et al. "Constraining depth range of $S$ wave velocity decrease after large 899 earthquakes near Parkfield, California." Geophysical Research Letters 43.12 (2016): 61296136.

903

Zaccarelli, Lucia, et al. "Variations of crustal elastic properties during the 2009 L'Aquila 904 earthquake inferred from cross-correlations of ambient seismic noise." Geophysical Research

905

906

907

908

909 